\title{
ON MULTIDIMENSIONAL BICM-ID CONSTELLATION LABELLING
}

\author{
O. Alamri ${ }^{1}$, B. Poupart ${ }^{2}$, M. El-Hajjar ${ }^{2}, S-X . \mathrm{Ng}^{2}$ and L. Hanzo ${ }^{2}$ \\ ${ }^{1}$ Department of Electrical Engineering, University of Umm Al-Qura, Saudi Arabia. \\ ${ }^{2}$ School of Electronics and Computer Science, University of Southampton, SO17 1BJ, UK. \\ Tel: +44-2380-593 125, Fax: +44-2380-593 045 \\ http: //www-mobile.ecs.soton.ac.uk
}

\begin{abstract}
It is widely recognised that the choice of a specific bit-to-symbol mapping scheme or a beneficial constellation labelling is an influential factor, when designing bit-interleaved coded modulation using iterative decoding (BICM-ID) for the sake of achieving a high iteration gain. Generally, a larger constellation size of a higher-dimensional space renders the bit-to-symbol mapping design more flexible. In this paper, we first define a flexibility measure based on the extrinsic information transfer (EXIT) characteristics of bit-to-symbol mapping schemes. Secondly, we investigate the effect of expanding the constellation to a higher-dimensional space on our proposed EXIT characteristic flexibility index, when transmitting diverse numbers of bits per joint symbol.
\end{abstract}

\section{INTRODUCTION}

The bit-error ratio (BER) performance curves of bit-interleaved coded modulation using iterative decoding (BICM-ID) may be divided into three regions: 1) the region of low signal-to-noise ratio (SNR) with negligible iterative BER reduction; 2) the so-called turbo-cliff or waterfall region exhibiting persistent iterative BER reduction over many iterations; and finally 3 ) the BER floor region typically experienced at moderate to high SNRs, where a rather low BER can be reached after a few iterations [1]. In order to attain a near-capacity performance, the SNR value corresponding to the turbo cliff has to be reduced. The crucial point at the receiver is that the concatenated detectors/demappers/decoders are soft-in/soft-out (SISO) decoders that accept and deliver probabilities or soft values, where the extrinsic information of the softoutput of one detector/demapper/decoder is passed on to the other detector/demapper/decoder to be used as a priori input.

Consequently, in [2], the employment of the turbo principle [3] was considered for iterative soft demapping in the context of BICMID, where a soft demapper was used between the multilevel demodulator and the channel decoder. Since then, it was widely recognised that the choice of a specific bit-to-symbol mapping scheme or a beneficial constellation labelling is an influential factor, when designing BICM-ID schemes exhibiting a high iteration gain [4-8]. In [4] ten Brink advocated the construction of different bit-to-symbol mapping schemes based on the bitwise mutual information, whereas the mapping schemes designed in [7] was based on the so-called Binary Switching Algorithm (BSA), which was previously employed for codebook index assignment optimisation in vector quantisation [9]. Recently, it was shown in [8] that the constellation design problem may be viewed as Quadratic Assignment Problem (QAP) [10], which may be optimised using the powerful Reactive Tabu Search (RTS) technique of [11].

The financial support of the European Community under Seventh Framework Programme grant agreement ICT OPTIMIX nINFSO-ICT214625 and of the EPSRC UK is gratefully acknowledged.
Generally, a larger constellation size of a higher-dimensional space renders the bit-to-symbol mapping design more flexible. Multi-dimensional constellations were shown to be beneficial in the design of TCM schemes as early as 1987 [12,13]. Additionally, multi-dimensional labelling was also proposed for QPSK based BICM-ID for transmission over a single antenna [14]. Further improvements of multi-dimensional constellation labelling were proposed in $[15,16]$. More recently, multi-dimensional constellation labelling was also proposed for bit-interleaved space-time-coded modulation using iterative decoding in [17], where the labelling of the two 16-QAM symbols transmitted over two antennas in two consecutive time-slots was designed jointly and was optimised using the RTS technique [11].

However, the above-mentioned contributions [14-17] do not provide a quantitative definition of the flexibility attained by the proposed bit-to-symbol mapping schemes in a higher-dimensional space. In addition, it is not clear, whether the achievable flexibility is due to expanding the constellation to a higher-dimensional space or accrues simply from increasing the number of bits per joint symbol. The contribution of this paper is twofold. Firstly, we define a quantitative flexibility measure based on the extrinsic information transfer (EXIT) characteristics [4] of diverse bit-to-symbol mapping schemes, where the employment of the EXIT characteristics between a concatenated decoder's input and output allows us to characterise the flow of extrinsic information through the softin/soft-out constituent decoders. Secondly, we quantify the effects of increasing the number of bits per joint symbol on our proposed EXIT-based flexibility index, when expanding the constellation to a higher-dimensional space.

This paper is organised as follows. A brief description of our BICM-ID system is presented in Section 2. In Section 3 we describe our proposed EXIT-based flexibility index. Our simulation results and discussions are provided in Section 4. Finally, we conclude in Section 5.

\section{SYSTEM OVERVIEW}

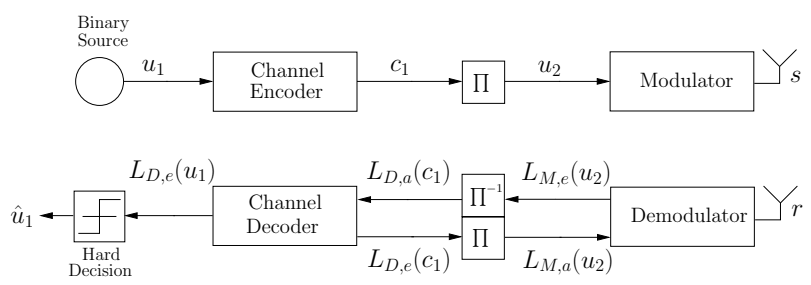

Figure 1: The block diagram of a BICM-ID system.

The schematic of the entire system is shown in Figure 1, where the transmitted source bits $\mathbf{u}_{1}$ are encoded by the channel encoder. 
This paper considers a $1 / 2$-rate recursive systematic convolutional (RSC) code. The channel encoded bits $\mathbf{c}_{1}$ are then interleaved by a random bit interleaver, producing the permuted bits $\mathbf{u}_{2}$. After channel interleaving, the $M$-ary modulator maps blocks of $n \cdot B$ channel-coded and interleaved bits to $n$ consecutive complexvalued $M$-ary symbols $\mathbf{s}=\left[s_{1}, \ldots, s_{n}\right]$, where $B=\log _{2} M$. There are $M^{n}$ distinct combinations of the $n$ consecutive complexvalued $M$-ary symbols that the modulator can choose from. The $n$ consecutive symbols can be represented in the $2 n$-dimensional real-valued Euclidean space $\mathbb{R}^{2 n}[18]$ as follows: $\mathbf{s}=\left[s_{1}, \ldots, s_{n}\right]$ $=\left[a_{1}, a_{2}, \ldots, a_{2 n}\right]$, where $s_{i}=a_{2 i-1}+j a_{2 i}$ represents the $i$-th complex-valued $M$-ary symbol.

In this treatise, we considered an uncorrelated narrowband Rayleigh fading channel, based on Jakes' fading model [19]. Two cases are considered: (1) The complex-valued fading envelope $h$ is assumed to be constant across the transmission period of $n$ consecutive complex-valued $M$-ary symbols spanning $T=n$ time slots; and (2) The complex-valued fading envelope $h$ is assumed to be different for each complex-valued $M$-ary symbol. The first case is referred to here as simultaneous fading, while the second case is referred to as independent fading. The complex Additive White Gaussian Noise (AWGN) of $w=w_{I}+j w_{Q}$ is also added to the received signal, where $w_{I}$ and $w_{Q}$ are two independent zero-mean Gaussian random variables having a variance of $\sigma_{w}^{2}=\sigma_{w_{I}}^{2}=\sigma_{w_{Q}}^{2}=N_{0} / 2$ per dimension, where $N_{0} / 2$ represents the double-sided noise power spectral density expressed in $W / H z$. Accordingly, the received signal corresponding to the $2 n$-dimensional transmitted symbol $\mathbf{s}=\left[s_{1}, \ldots, s_{n}\right]$ may be represented by $\mathbf{r}=\left[r_{1}, \ldots, r_{n}\right]$, where $r_{i}=h_{i} s_{i}+w_{i}, i=1, \ldots, n$.

As shown in Figure 1, each $2 n$-dimensional symbol $\mathbf{r}$ represents a block of $n \cdot B$ coded bits. Assuming that the complexvalued fading envelope $h$ is perfectly known at the receiver, iterative demapping/decoding is carried out between the demodulator and the APP-based SISO channel decoder, where extrinsic information is exchanged between the constituent demapper/decoder modules $[2,20]$ More specifically, $L_{\cdot, a}(\cdot)$ in Figure 1 represents the a priori information, expressed in terms of the log-likelihood ratios (LLRs) of the corresponding bits, whereas $L_{., e}(\cdot)$ represents the extrinsic LLRs of the corresponding bits. The iterative process is performed for a number of consecutive iterations. During the last iteration, only the LLR values $L_{I, e}\left(\mathbf{u}_{\mathbf{1}}\right)$ of the original uncoded systematic information bits $\mathbf{u}_{1}$ are required, which are passed to a hard decision decoder in order to determine the estimated transmitted source bits $\hat{\mathbf{u}}_{1}$, as shown in Figure 1.

\section{EXIT CHART ANALYSIS}

\subsection{Preliminaries}

The main objective of employing EXIT charts [4], is to predict the convergence behaviour of the iterative decoder by examining the evolution of the input/output mutual information exchange between the inner and outer decoders in consecutive iterations. The application of EXIT charts is based on the two assumptions, namely that upon assuming large interleaver lengths, (1) the $a$ prior $i$ LLR values are fairly uncorrelated; (2) the a priori $i$ LLR values exhibit a Gaussian PDF.

Let $I_{, a}(x), 0 \leq I_{,, a}(x) \leq 1$, denote the mutual information between the a priori LLRs $L_{,, a}(x)$ as well as the corresponding bits $x$ and let $I_{,, e}(x), 0 \leq I_{,, e}(x) \leq 1$, denote the mutual information between the extrinsic LLRs $L_{\cdot, e}(x)$ and the corresponding bits $x$, where the subscript $(\cdot)$ is used to distinguish the channel decoder (D) and the demodulator (M).

\subsection{Extrinsic Information Transfer Chart}

The exchange of extrinsic information in the system schematic of Figure 1 is visualised by plotting the extrinsic information transfer characteristics of the demodulator and of the outer RSC decoder in a joint diagram. This diagram is known as the Extrinsic Information Transfer (EXIT) chart [4]. The outer RSC decoder's extrinsic output $I_{D, e}\left(c_{1}\right)$ becomes the demodulator's a prior $i$ input $I_{M, a}\left(u_{2}\right)$, which is represented on the $x$-axis. Similarly, on the $y$-axis, the demodulators's extrinsic output $I_{M, e}\left(u_{2}\right)$ becomes the outer RSC decoder's a priori input $I_{D, a}\left(c_{1}\right)$. For the rest of the paper $I_{D, \cdot}\left(c_{1}\right)$ and $I_{M, \cdot}\left(u_{2}\right)$ will be simply denoted by $I_{D, \cdot}$ and $I_{M, .}$, respectively.

Figure 2 shows the EXIT chart of a BICM-ID scheme employing QPSK modulation and a 1/2-rate RSC code having octally represented generator polynomials of $\left(G_{r}, G\right)=(7,5)_{8}$, where $G_{r}$ is the feedback polynomial. Figure 2 illustrates the EXIT chart of the system, when operating at $E_{b} / N_{0}=5 \mathrm{~dB}$ and communicating over the uncorrelated Rayleigh fading channel considered. Ideally, in order for the exchange of extrinsic information between the demodulator and the outer RSC decoder to converge at a specific $E_{b} / N_{0}$ value, the EXIT curve of the demodulator recorded at the $E_{b} / N_{0}$ value of interest and that of the outer RSC decoder should only intersect at a point infinitesimally close to the $I_{D, e}=1.0$ line. If this condition is satisfied, then a so-called convergence tunnel [4] appears in the EXIT chart. The narrower the tunnel, the more iterations are required for reaching the point of intersection.

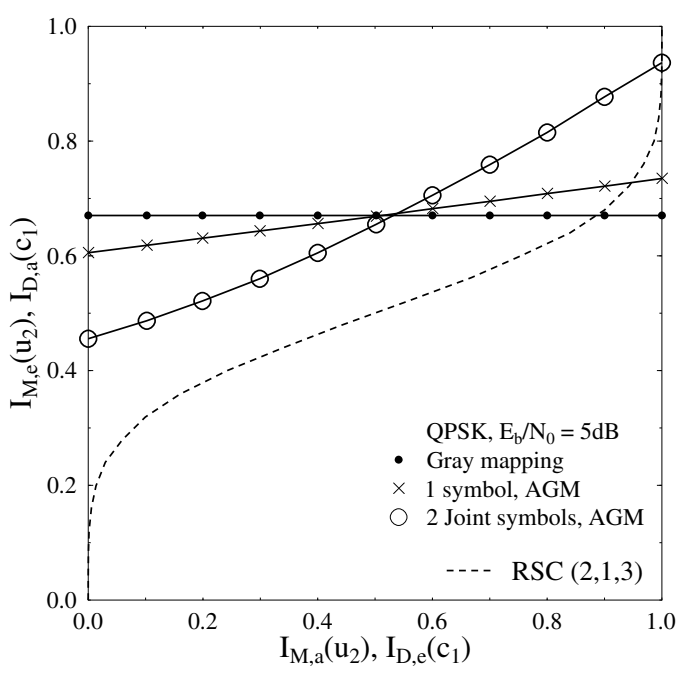

Figure 2: EXIT chart of a QPSK-modulated BICM-ID scheme employing Gray mapping $(\mathrm{GM})$ and anti-Gray mapping (AGM) in combination with a $1 / 2$-rate RSC code, when communicating over an uncorrelated Rayleigh fading channel.

As expected, Gray mapping (GM) does not provide any iteration gain upon increasing the mutual information at the input of the demodualtor [1]. However, using anti-Gray mapping (AGM) ${ }^{1}$ schemes [2] results in beneficial iteration gain, upon increasing the mutual information, as illustrated by the positive slopes of the EXIT curves of the AGM schemes seen in Figure 2. The AGM schemes shown in Figure 2 were obtained by invoking the RTS al-

\footnotetext{
${ }^{1}$ Any bit-to-symbol mapping scheme, which is different from classic Gray mapping is termed as an AGM scheme, as detailed in [1].
} 
gorithm $[8,11]$ to minimise the following error-bound based cost function [7]

$$
C F_{e b}=\frac{1}{B 2^{B}} \sum_{i=1}^{B} \sum_{b=0}^{1} \sum_{s_{k} \in \chi_{b}^{i}} \sum_{\hat{s}_{k} \in \chi_{b}^{i}} \frac{1}{\left|s_{k}-\hat{s}_{k}\right|^{2}},
$$

where $\chi_{b}^{i}$ denotes the subset of symbols $s_{k} \in \chi$ whose bit labels have the value of $b \in\{0,1\}$ in position $i \in\{1, \ldots, B\}^{2}$. Observe in Figure 2 that when detecting two QPSK symbols jointly, the attainable iteration gain achieved upon increasing the mutual information becomes higher than in case of considering each QPSK symbol separately. The higher iteration gain is represented by the steeper slope of the EXIT curve in Figure 2. This implies that according to the predictions of the EXIT chart seen in Figure 2, the iterative decoding process is expected to converge to a lower BER at $E_{b} / N_{0}=5.0 \mathrm{~dB}$, when considering a pair of QPSK symbols jointly, as compared to the separate decoding and demapping of the QPSK symbols.

These EXIT chart based convergence predictions are verified by the actual BER performance depicted in Figure 3, which compares the performance of the BICM-ID system employing GM and AGM schemes in conjunction with QPSK modulation and the RSC code of Figure 2, when communicating over an uncorrelated Rayleigh fading channel and employing an interleaver depth of $D=10^{5}$ bits. Observe in Figure 3 by comparing the two GMbased BICM-ID curves that as expected no BER improvement was obtained, when five turbo-detection iterations were employed in conjunction with GM. This phenomenon was reported also in [2] and becomes evident from the horizontal curve of the GM seen in Figure 2. By contrast, AGM achieved a useful performance improvement in conjunction with iterative demapping and decoding. Moreover, as predicted from the EXIT chart of Figure 2, the joint demapping and decoding of two QPSK symbols provided further BER performance improvements compared to the separate demapping and decoding of QPSK symbols.

\subsection{EXIT Chart Flexibility Index}

The discussion presented in Section 3.2 highlights two important observations; namely that (i) a steeper EXIT curve slope translates to an improved BER performance; (ii) the joint demapping and decoding of multiple modulated symbols increases the slope of the AGM EXIT curves. Accordingly, the benefits and flexibility offered by the bit-to-symbol mapping schemes in a higherdimensional space [14-17] may be linked to and hence represented by the slope of the AGM EXIT curve. For example, Figure 4-a shows both the GM and AGM EXIT curves of a BICM-ID schemes employing 16QAM modulation and operating at $E_{b} / N_{0}=5.0 \mathrm{~dB}$. The additional EXIT-chart flexibility offered by the AGM schemes compared to the corresponding GM scheme may be quantified by the gray area in Figure 4-a, which represents the deviation of the AGM EXIT curve from the corresponding GM EXIT curve. This flexibility measure is referred to here as the EXIT flexibility index $F_{I}$. The gray area in Figure 4-a can be measured by numerical integration. However, since the demapper's EXIT characteristic

${ }^{2}$ For example, consider a QPSK constellation consisting of four symbols, where each symbol represents two binary bits $\left(b_{2} b_{1}\right)$ as follows: $S 0 \mapsto 00, S 1 \mapsto 01, S 2 \mapsto 10$ and $S 3 \mapsto 11$. Then, the cost function of Equation (1) becomes

$$
\begin{aligned}
C F_{e b}= & \frac{1}{8}\left(\frac{2}{|S 0-S 1|^{2}}+\frac{2}{|S 0-S 2|^{2}}+\frac{4}{|S 0-S 3|^{2}}\right. \\
& \left.+\frac{4}{|S 1-S 2|^{2}}+\frac{2}{|S 1-S 3|^{2}}+\frac{2}{|S 2-S 3|^{2}}\right),
\end{aligned}
$$

where the value of $C F_{e b}$ depends on the complex-valued representations of $S 0, S 1, S 2$ and $S 3$, which is basically the mapping scheme.

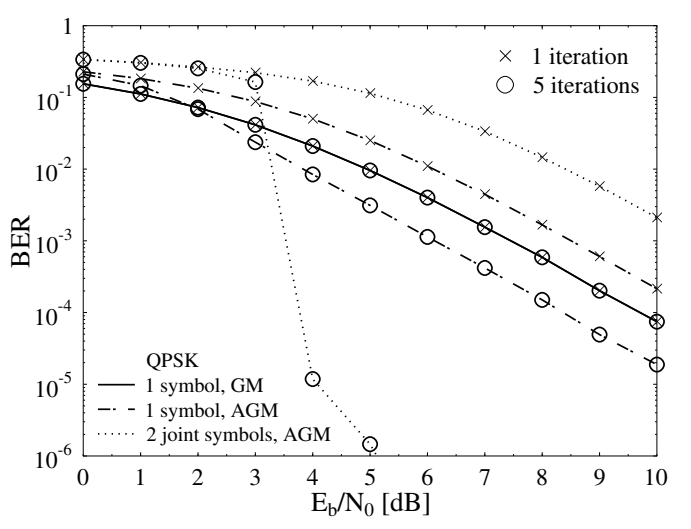

Figure 3: Performance comparison of the BICM-ID system employing GM and AGM schemes in conjunction with QPSK modulation and a 1/2-rate RSC code, when communicating over an uncorrelated Rayleigh fading channel and employing an interleaver depth of $D=10^{5}$ bits.

curves are sufficiently smooth, the gray area in Figure 4-a may be conveniently approximated by the distances shown in Figure 4-b, which may be evaluated for the SNR range of our interest as follows. Considering $I_{M, e}$ as a function of both $I_{M, a}$ and the $E_{b} / N_{0}$ value encountered, the demapper's EXIT characteristic is defined as $I_{M, e}=T_{M}\left(I_{M, a}, E_{b} / N_{0}\right)$ [4]. Now, the EXIT flexibility in$\operatorname{dex} F_{I}$ may be formulated as

$$
F_{I}=\frac{1}{N} \sum_{i=1}^{N} \sum_{I_{M, a}=0}^{1}\left|T_{M}^{A G M}\left(I_{M, a}, \rho_{i}\right)-T_{M}^{G M}\left(I_{M, a}, \rho_{i}\right)\right|
$$

where $T_{M}^{A G M}(\cdot)$ and $T_{M}^{G M}(\cdot)$ refer to the EXIT characteristics of the AGM and GM schemes, respectively. Moreover, $\rho_{i}, 1 \leq i \leq$ $N$, represents the specific $E_{b} / N_{0}$ values of interest. (a)

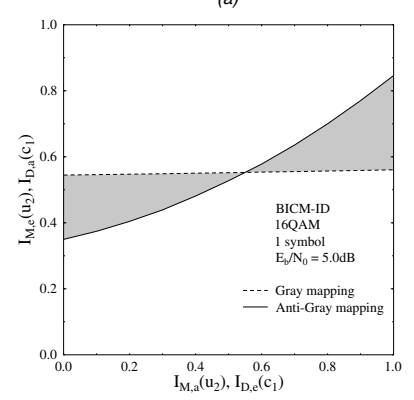

(b)

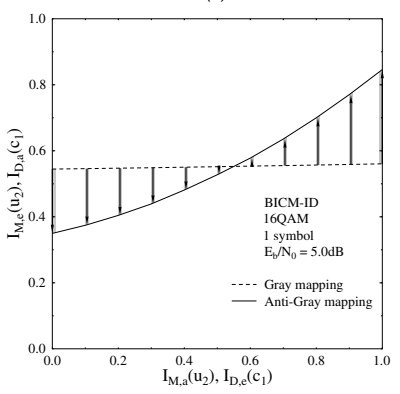

Figure 4: EXIT flexibility index $F_{I}$. (a) Area between the AGM EXIT curve and the corresponding GM EXIT curve. (b) Distance-based approximation of $F_{I}$.

In order to determine the appropriate SNR range to be considered when calculating $F_{I}$, it is essential to understand the influence of increasing the SNR on the EXIT characteristics of the demodulator. Figure 5-a shows the extrinsic information $I_{M, e}$ at the output of the demodulator of the BICM-ID scheme of Figure 1, when employing GM-based 16QAM and operating from $E_{b} / N_{0}=-8.0 \mathrm{~dB}$ to $E_{b} / N_{0}=24.0 \mathrm{~dB}$. Observe in Figure 5-a that the extrinsic information $I_{M, e}$ at the output of the demodulator exhibits the fastest improvement upon increasing the SNR in steps of $0.5 \mathrm{~dB}$ across a specific range of $E_{b} / N_{0}$ values. Figure 5-b 
highlights this phenomenon by illustrating the improvement of the extrinsic information $I_{M, e}$ at the output of the demodulator upon increasing the SNR, when no a priori information is available at the input of the demodulator (i.e. we have $I_{M, a}=0$ ). It is evident from Figure 5-b that there is a specific range of SNR values for which the improvement of $I_{M, e}$ is the fastest as a function of $E_{b} / N_{0}$. This area is referred to as the linear region of mutual information dependence on the $E_{b} / N_{0}$ in Figure 5-b. This linear region is of special interest to us, because it corresponds to the turbo-cliff area in the BER performance curve mentioned in Section 1, given a specific outer channel decoder, excluding outer decoders having either extremely low code rate (i.e. $R<0.2$ ) or extremely high code rate (i.e. $R>0.8$ ).

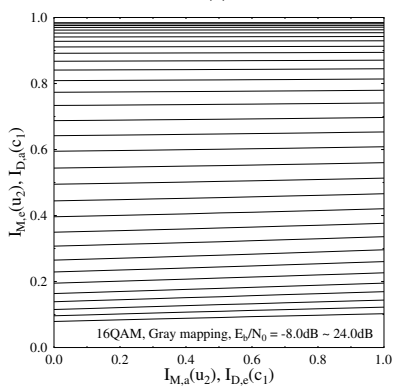

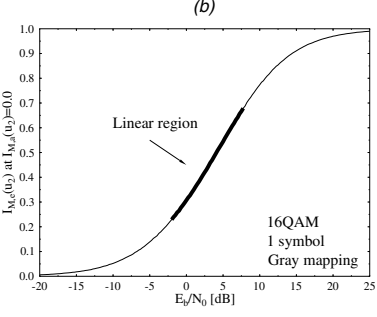

Figure 5: (a) Extrinsic information $I_{M}$ at the output of the demodulator of the BICM-ID scheme of Figure 1, when employing GM-based 16QAM and increasing the SNR in $0.5 \mathrm{~dB}$ steps from $E_{b} / N_{0}=-8.0 \mathrm{~dB}$ to $E_{b} / N_{0}=24.0 \mathrm{~dB}$. (b) The improvement of the extrinsic information $I_{M, e}$ at the output of the demodulator upon increasing the SNR, when no a priori information is available at the input of the demodulator

\section{RESULTS AND DISCUSSIONS}

Having defined the EXIT flexibility index in Section 3.3, which provides a quantitative criterion for comparing different multidimensional constellation labelling schemes, we embark on addressing the second contribution of this paper. More specifically, various multidimensional modulation constellation schemes are studied in order to investigate the effect of expanding the constellation to a higher-dimensional space as well as to quantify the influence of increasing the number of bits per joint symbol on our proposed EXIT-based flexibility index. For the sake of calculating the EXIT flexibility index $F_{I}$, all AGM schemes considered in this section were obtained so that the cost function in Equation (1) is optimised by invoking the RTS algorithm [8,11], which always obtains the global optimimum solution. Moreover, for the various multidimensional modulation constellation schemes considered in this section, the EXIT flexibility index $F_{I}$ was determined for specific SNR ranges that are associated with the corresponding linear region described in Section 3.3.

Figure 6 shows the effect of the joint decoding of multiple symbols on the achievable EXIT flexibility index $F_{I}$, for three modulation types, namely for QPSK, 8PSK and 16QAM. Furthermore, Figure 6 also illustrates the influence of the two different channel fading scenarios considered on the achievable $F_{I}$, where the case of simultaneous fading is characterised in Figure 6a, while that of independent fading is shown in Figure 6-b. Both of these channel fading senarii were defined in Section 2. Observe in Figure 6-a and Figure 6-b that a useful EXIT flexibility improvement may be achieved, when decoding multiple symbols jointly. However, the improvement beyond two jointly decoded symbols becomes incremental for the case of QPSK modulation.
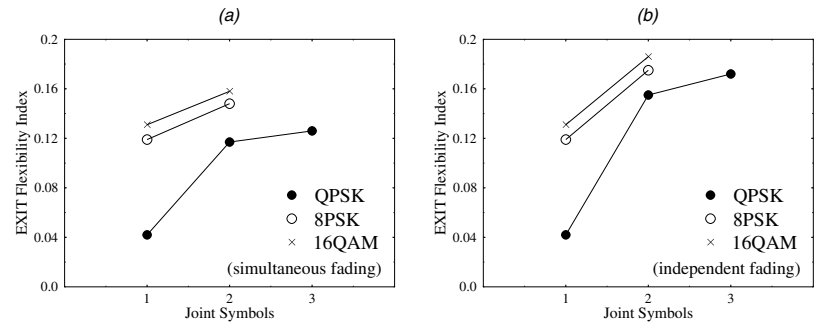

Figure 6: EXIT flexibility index $F_{I}$, when considering various number of joint symbols and different modulation schemes. (a) The case of a simultaneous fading based Rayleigh channel model. (b) The case of an independent fading based Rayleigh channel model.

Figure 7-a demonstrates the influence of employing different modulation schemes on the attainable EXIT flexibility index $F_{I}$. It is evident from Figure 7-a that the EXIT flexibility index $F_{I}$ improves upon increasing the number of bits-per-symbol. Moreover, decoding two symbols jointly provides a better EXIT flexibility improvement, when communicating over an independent fading based Rayleigh channel as compared to a simultaneous fading based Rayleigh channel. Observe furthermore in Figure 7-a that the joint decoding of two symbols corresponds to doubling the number of bits-per-joint-symbol, which corresponds to the additional decoding complexity illustarted in Figure 7-b in terms of the size of search space per bit and expressed in units of [symbols/bit]. The decoding complexity quantified in Figure 7-b increases exponentially with the number of jointly detected symbols, when computing the LLR values of each bit. More specifically, in this figure a quadratic increase is observed for two joint symbols. However, it cannot be explicitly inferred from Figure 7-a, whether the EXIT flexibility improvement is due to the decoding of two joint symbols or due to the increase of the number of bits-per-symbol. Subsequently, Figure 8-a shows the effect of the number of bits-perjoint-symbol on the achievable EXIT flexibility index $F_{I}$, when expanding the modulation constellation from two dimensions to four dimensions, where the various modulation constellations are defined in Table 1. Figure 8-a demonstrates that the EXIT flexibility improvement shown in Figure 7-a, when decoding two symbols jointly and communicating over a simultaneous fading-based Rayleigh channel, was mainly due to the increase in the number of bits-per-joint-symbol. However, when communicating over an independent fading-based Rayleigh channel, expanding the constellation to four dimensions offers further EXIT flexibility improvements compared to the conventional 2-dimensional constellations and for a given number of bits-per-joint-symbol.
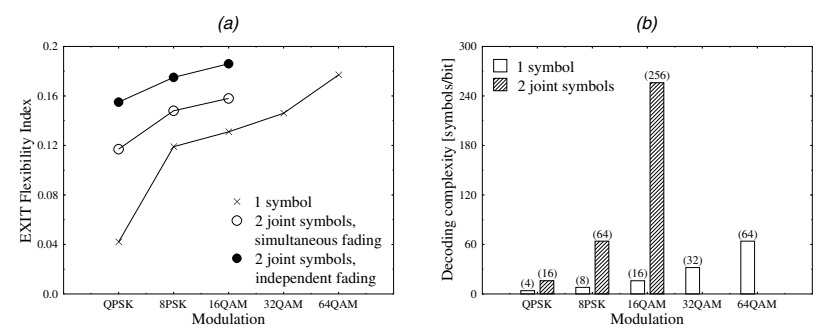

Figure 7: (a) EXIT flexibility index $F_{I}$, when considering different modulation schemes as well as single-symbol decoding and the decoding of two joint symbols, when communicating over simultaneous and independent fading based Rayleigh channels. (b) Complexity of schemes in (a). 


\begin{tabular}{|c|l|l|}
\hline Block Size & \multicolumn{2}{|c|}{ Dimensions of the Constellation } \\
\cline { 2 - 3 } Size & \multicolumn{1}{|c|}{ 2 Dimensions } & \multicolumn{1}{c|}{ 4 Dimensions } \\
\hline \hline 2 & one QPSK symbol & - \\
\hline 3 & one 8-PSK symbol & - \\
\hline 4 & one 16-QAM symbol & two joint QPSK symbols \\
\hline 5 & one 32-QAM symbol & - \\
\hline 6 & one 64-QAM symbol & two joint 8-PSK symbols \\
\hline 8 & - & two joint 16-QAM symbols \\
\hline
\end{tabular}

Table 1: Modulation schemes corresponding to various block sizes of bits-perjoint-symbol.
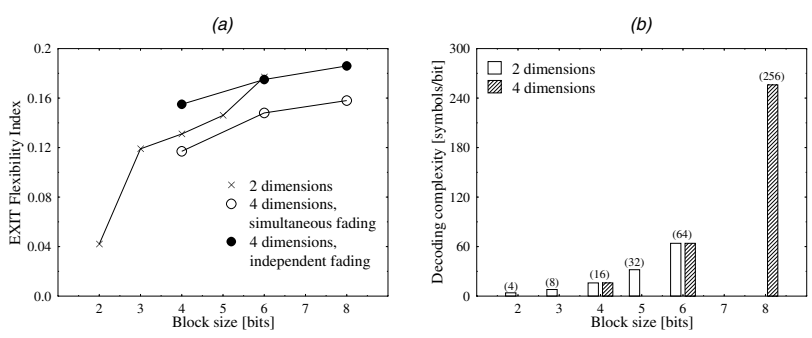

Figure 8: (a) EXIT flexibility index $F_{I}$, when considering different number of bits per joint symbol as well as expanding the modulation constellation from two dimensions to four dimensions for transmission over simultaneous and independent fading based Rayleigh channels. (b) Complexity of schemes in (a).

\section{CONCLUSION}

We investigated multidimensional BICM-ID constellation labelling. We first proposed a flexibility measure based on the EXIT characteristics of diverse bit-to-symbol mapping schemes, which was referred to as the EXIT flexibility index. We also investigate the effect of expanding the constellation to a higher-dimensional space as well as the beneficial influence of increasing the number of bits per joint symbol on our proposed EXIT-based flexibility index. It was observed that the EXIT flexibility improvement offered by expanding the modulation constellation to a higher-dimensional space, when communicating over a simultaneous fading based Rayleigh channel was mainly due to the increased number of bitsper-joint-symbol. However, useful EXIT flexibility improvements may also be obtained by expanding the modulation constellation to a higher-dimensional space, especially when communicating over an independent fading based Rayleigh channel.

\section{REFERENCES}

[1] L. Hanzo, O. Alamri, M. Elhajjar and N. Wu, Near-Capacity Multi-Functional MIMO Systems: Sphere-Packing, Iterative Detection and Cooperation. Chichester, England: John Wiley and Sons, 2009 (in press).

[2] S. ten Brink, J. Speidel and R.-H. Yan, "Iterative demappping and decoding for multilevel modulation," in Procedings of IEEE Global Telecommunications Conference (GLOBECOM), vol. 1, (Sydney, Australia), pp. 579-584, November 1998.

[3] C. Berrou, A. Glavieux and P. Thitimajshima, "Near Shannon Limit Error-Correcting Coding and Decoding: Turbo Codes," in IEEE International Confrence on Communications, (Geneva, Switzerland), pp. 1064-1070, May 1993.

[4] S. ten Brink, "Designing iterative decoding schemes with the extrinsic information transfer chart," $A E \ddot{U}$ International Journal of Electronics and Communications, vol. 54, pp. 389-398, November 2000.
[5] A. Chindapol and J. A. Ritcey, "Design, analysis, and performance evaluation for BICM-ID with square QAM constellations in Rayleigh fading channels," IEEE Journal on Selected Areas in Communications, vol. 19, pp. 944-957, May 2001.

[6] J. Tan and G. L. Stüber, "Analysis and design of symbol mappers for iteratively decoded BICM," IEEE Transactions on Wireless Communications, vol. 4, pp. 662-672, March 2005.

[7] F. Schreckenbach, N. Görtz, J. Hagenauer and G. Bauch, "Optimization of symbol mappings for bit-interleaved coded Modulation with iterative decoding," IEEE Communications Letters, vol. 7, pp. 593-595, December 2003.

[8] Y. Huang and J. A. Ritcey, "Improved 16-QAM constellation labeling for BI-STCM-ID with the Alamouti scheme," IEEE Communications Letters, vol. 9, pp. 157-159, February 2005.

[9] K. Zeger and A. Gersho, "Pseudo-Gray coding," IEEE Transactions on Communications, vol. 38, pp. 2147-2158, December 1990.

[10] E. Cela, The Quadratic Assignment Problem: Theory and Algorithms. Norwell, MA: Kluwer Academic, 1998.

[11] R. Battiti and G. Tecchiolli, "Reactive search: toward selftuning heuristics," Operations Research Society of America (ORSA) journal on computing, vol. 6, no. 2, pp. 126-140, 1994.

[12] L.-F. Wei, "Trellis-coded modulation with multidimensional constellations," IEEE Transactions on Information Theory, vol. 33, pp. 483-501, July 1987.

[13] S. S. Pietrobon, R. H. Deng, A. Lafanechere, G. Ungerboeck and D. J. Costello-Jr., "Trellis-coded multidimensional phase modulation," IEEE Transactions on Information Theory, vol. 36, pp. 63-89, January 1990.

[14] N. H. Tran and H. H. Nguyen, "Design and performance of BICM-ID systems with hypercube constellations," IEEE Transactions on Wireless Communications, vol. 5, pp. 11691179, May 2006.

[15] S. Bäro, "Turbo-detection in MIMO systems: bit labeling and pre-coding," in 5th International ITG Conference on Source and Channel Coding, (Erlangen, Germany), January 2004.

[16] F. Simoens, H. Wymeersch, H. Bruneel and M. Moeneclaey, "Multidimensional mapping for bit-interleaved coded modulation with BPSK/QPSK signaling," IEEE Communications Letters, vol. 9, pp. 453-455, May 2005.

[17] A. S. Mohammed, W. Hidayat and M. Bossert, "Multidimensional 16-QAM constellation labeling of BI-STCM-ID with the Alamouti scheme," in Proceedings of IEEE Wireless Communications and Networking Conference (WCNC), (Las Vegas, USA), April 2006.

[18] J. H. Conway and N. J. Sloane, Sphere Packings, Lattices and Groups. Springer-Verlag, 1999.

[19] W. C. Jakes, Microwave Mobile Communications. New York: Wiley, 1974.

[20] S. Benedetto, D. Divsalar, G. Montorsi and F. Pollara, "A soft-input soft-output APP module for iterative decoding of concatenated codes," IEEE Communications Letters, vol. 1, pp. 22-24, January 1997. 